\title{
Multiple species of arsenic biotransformation occur in Nostoc sp. PCC 7120
}

\author{
X.M. Xue \& Y.G. Zhu \\ Key Lab of Urban Environment and Health, Institute of Urban Environment, \\ Chinese Academy of Sciences, Xiamen, China
}

\begin{abstract}
Nostoc cells incubated with arsenite (As(III)) for two weeks were extracted with dichloromethane/methanol (DCM/MeOH) and the extract was partitioned between water and DCM. Arsenic species in aqueous and DCM layers were determined using high performance liquid chromatography - inductively coupled plasma mass spectrometer/electrospray tandem mass spectrometry (HPLC-ICPMS/ESIMSMS). In addition to inorganic arsenic (iAs), the aqueous layer also contained monomethylarsonate (MAs(V)), dimethylarsinate (DMAs(V)), and the two arsenosugars, namely a glycerol arsenosugar (Oxo-Gly) and a phosphate arsenosugar $\left(\mathrm{Oxo}-\mathrm{PO}_{4}\right)$. Two major arsenosugar phospholipids (AsSugPL982 and AsSugPL984) were detected in DCM fraction. Arsenic in the growth medium was also investigated by HPLC/ICPMS and shown to be present mainly as the inorganic forms As(III) and As(V) accounting for $29 \%-38 \%$ and $29 \%-57 \%$ of the total arsenic, respectively. The total arsenic of methylated arsenic, arsenosugars, and arsenosugar phospholipids in Nostoc cells with increasing As(III) exposure were not markedly different, indicating that the transformation to organoarsenic in Nostoc was not dependent on As(III) concentration in the medium.
\end{abstract}

\section{INTRODUCTION}

Arsenic (As) is a ubiquitous and carcinogenic toxic element, and has both acute and chronic toxicity effects on humans. The bioavailability of arsenic and its resultant toxicity are influenced to a great extent by its species. ${ }^{1}$ Inorganic arsenic, the major form of arsenic in water and soils, is transformed into organic arsenic species or in reverse in natural biological processes, and microorganisms play a critical role in arsenic biogeochemical cycle (Zhu et al., 2014).

Cyanobacteria are involved in arsenic biogeochemical cycle, and have been reported to have the ability to methylate inorganic arsenic, producing arsenosugars and arsenosugar phospholipids. The previous studies showed that Nostoc methylated As(III) to DMAs(V) and TMA(O) (Yin et al., 2011), demethylated MAs(V) and MAs(III) into As(III) (Yan et al., 2015), and produced Oxo-Gly (Miyashita et al., 2012). In this study, HPLC-ICPMS/ESIMS was used to analyze arsenic biotransformation in Nostoc in order to understand arsenic biotransformation by Nostoc from multiple perspectives.

\section{METHODS/EXPERIMENTAL}

\subsection{Fractionation of arsenic in Nostoc}

About $30 \mathrm{mg}$ of freeze-dried cells were weighed (to a precision of $0.1 \mathrm{mg}$ ) directly into a centrifuge tube $(15 \mathrm{~mL}$, polypropylene), $5 \mathrm{~mL}$ of a mixture of $\mathrm{DCM} / \mathrm{MeOH}(2+1, \mathrm{v} / \mathrm{v})$ was added. The mixture was extracted on a rotary wheel overnight, and centrifuged at $4754 \mathrm{~g}$ and $4{ }^{\circ} \mathrm{C}$ for 15 minutes. $0.5 \mathrm{~mL}$ of $1 \%$ aqueous $\mathrm{NH}_{4} \mathrm{HCO}_{3}$ solution was added to the supernatant $(\sim 4.5 \mathrm{~mL})$, the solution was separated into an aqueous layer (upper layer, $\mathrm{MeOH}$ and $\mathrm{H}_{2} \mathrm{O}$ ) and $\mathrm{DCM}-\mathrm{MeOH}$ layer (lower layer).

\subsection{HPLC-ICPMS/ESIMSMS analysis of water-soluble arsenic species and arsenolipids}

Separation was performed under reversed-phase conditions using a Shodex Asahipak C8P-50 4D column $(4.6 \times 150 \mathrm{~mm}, 5 \mu \mathrm{m}$ particle size $)$. The column effluent was split using a passive splitter with $80 \%$ being transferred directly to the ESI-MSMS. The remaining $20 \%$ of the split flow was transferred to the ICPMS together with a support flow of water containing 1\% formic acid and $20 \mu \mathrm{g} \mathrm{L}^{-1} \mathrm{Ge}$, In, Te $\left(0.3 \mathrm{~mL} \mathrm{~min}^{-1}\right)$ introduced through a T-piece after the splitter.

\section{RESULTS AND DISCUSSION}

\subsection{Results}

Arsenic species in the aqueous layers were analyzed, and revealed that two arsenosugars were produced by Nostoc. The relative proportion of arsenosugars in total aqueous arsenic species in Nostoc cells was $0.5 \%-$ $13 \%$ for Oxo-Gly and $0.7 \%-8 \%$ for $\mathrm{Oxo}-\mathrm{PO}_{4}$, and decreased with increasing As(III) exposure.

HPLC/ICPMS analysis of the DCM fraction showed the presence of arsenolipids, and analysis by ESMSMS revealed that the $[\mathrm{M}+\mathrm{H}]^{+}$of two main arsenic-containing compounds were 983 and 985. The chromatographic behavior was the same as that reported previously for arsenosugar phospholipids 
$\left(\mathrm{C}_{47} \mathrm{H}_{88} \mathrm{O}_{14} \mathrm{AsP}\right.$ (As-PL982) and $\mathrm{C}_{47} \mathrm{H}_{90} \mathrm{O}_{14} \mathrm{AsP}$ (AsPL984) in Synechocystis sp. PCC 6803 and brown macroalgae.

\subsection{Discussion}

Multiple species of arsenic biotransformation pathways co-occur in Nostoc. Microbes have evolved various mechanisms to utilize or detoxify arsenic. Known mechanisms include arsenic redox changes, arsenic methylation and demethylation, As(III) efflux, and the production of complex organoarsenic. Arsenic reduction and efflux are always considered as significant arsenic detoxification in microorganisms. The previous proteomics analysis of Nostoc under $\operatorname{As}(\mathrm{V})$ stress showed that the expression of two genes, alr1097 (encoding an As(III) efflux protein; arsB) and alr1105 (encoding an $\mathrm{As}(\mathrm{V})$ reductase; $\operatorname{ars} C$ ), was up-regulated, (Pandey, et al., 2012), illustrating that Nostoc was capable of performing $\mathrm{As}(\mathrm{V})$ uptake, As(V) reduction, and As(III) excretion. Similar results were also found in cyanobacteria Synechocystis sp. PCC 6803 and Microcystis aeruginosa, some globally significant picocyanobacteria Prochlorococcus also were reported to have genomic potential for $\mathrm{As}(\mathrm{V})$ reduction and As(III) efflux. (Saunders \& Rocap 2015). Yin et al. (2011) investigated the ability of arsenic methylation by Nostoc and arsenic(III) S-adenosylmethionine methyltransferase (ArsM) from Nostoc by chemotrapping volatile TMAs(III). Moreover, our other study showed that Nostoc was able to demethylate MAs(III) rapidly to As(III) using ArsI that is a C.As lyase responsible for MAs(III) demethylation, and also could demethylate $\mathrm{MAs}(\mathrm{V})$ slowly to As(III), (Yan et al., 2015) suggesting that MAs(V) reduction and MAs(III) demethylation occurred in Nostoc. However, MAs(III) oxidation in Nostoc cannot be regarded to be catalyzed by an enzyme because ArsH homologous compound oxidizing MAs(III) (Chen, et al., 2015) was not found via blasting against the Nostoc proteome with ArsH of Synechocystis sp. PCC 6803, (Xue et al., 2014) and we did not ensure that there was As(III) oxidation catalyzed by As(III) oxidase in Nostoc because most of As(III) was oxidized by oxygen after long time culture and there was not As(III) oxidase identified in Nostoc. In addition, Nostoc was found to produce low quantities of arsenosugars and arsenolipids.

An abbreviated biosynthesis pathway of arsenosugar phospholipids in Nostoc was hypothesized as described in Figure 1. The methyl groups from S-adenosyl-L-methionine (SAM) are transferred to $\mathrm{As}(\mathrm{III})$ (which is absorbed directly by cells or arises from $\mathrm{As}(\mathrm{V})$ reduced by $\mathrm{Ars} C)$, by ArsM to produce trivalent methylated arsenic. Some of MAs(III) and DMAs(III) bound to ArsM are further methylated into DMAs(III) and TMAs(III). In addition, the adenosyl group from SAM is transferred to DMAs(III) falling off ArsM to generate the key intermediate of arsenosugar synthesis, namely dimethylarsinyladenosine (AsAd). AsAd undergoes glycosidation to produce Oxo-Gly which acts as a precursor to $\mathrm{Oxo}-\mathrm{PO}_{4}$

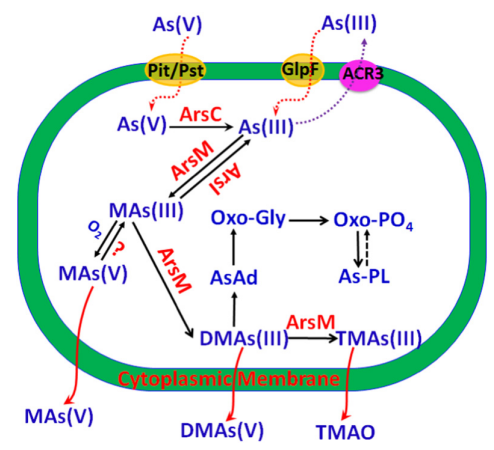

Figure 1. Arsenic metabolism and potential biosynthetic pathways of arsenosugar phospholipids in Nostoc.

produced later by cyanobacteria. Finally, fatty cids were added to $\mathrm{Oxo}-\mathrm{PO}_{4}$ to produce arsenosugar phospholipids by unknown enzymes.

In summary, our study revealed that the model organism cyanobacterium Nostoc can produce Oxo$\mathrm{PO}_{4}$ and arsenosugar phospholipids when exposed to $\mathrm{As}(\mathrm{III})$, and the production of complex organoarsenic and arsenic demethylation co-exist in Nostoc.

\section{ACKNOWLEDGEMENTS}

Our research is supported by the National Natural Science foundation of China (21507125).

\section{REFERENCES}

Chen, J., Bhattacharjee H., \& Rosen B.P. 2015. ArsH is an organoarsenical oxidase that confers resistance to trivalent forms of the herbicide monosodium methylarsenate and the poultry growth promoter roxarsone. Mol. Microbiol. 96(5): 1042-1052.

Miyashita, T., Oda Y., Horiuchi, J., Yin, J.C., Morimoto, T. \& Saitoe M. 2012. $\mathrm{Mg}^{2+}$ block of Drosophila NMDA receptors is required for long-term memory formation and CREB-dependent gene expression. Neuron. 74(5): 887-98.

Pandey, S., Rai, R. \& Rai, L.C. 2012. Proteomics combines morphological, physiological and biochemical attributes to unravel the survival strategy of anabaena Sp. PCC7120 under arsenic stress. J Proteomics. 75(3): 921-937.

Saunders, J. K. \& Rocap, G. 2015. Genomic potential for arsenic efflux and methylation varies among global prochlorococcus populations. The ISME Journal 10(1): 197-209.

Xue, X.M., Yan, Y., Xu, H.J., Wang, N., Zhang, X. \& Ye, J. 2014. ArsH from synechocystis Sp. PCC 6803 reduces chromate and ferric iron. FEMS Microbiol Letters 356 (1): 105-12.

Yan, Y., Ye, J., Xue, X.M. \& Zhu, Y.G. 2015. Arsenic demethylation by a C A As cyase in cyanobacterium nostoc Sp. PCC 7120. Environ. Sci. Technol. 49: 14350-58.

Yin, X.X., Chen, J., Qin, J., Sun, G.X., Rosen, B.P. \& Zhu Y.G. 2011. Biotransformation and volatilization of arsenic by three photosynthetic cyanobacteria. Plant Physiol. 156 (3): 1631-38.

Zhu, Y.G., Yoshinaga M., Zhao F.J. \& Rosen B.P. 2014. Earth abides arsenic biotransformations. Annu. Rev. Earth Plan. Sci. 42(1): 443-67. 